\title{
A note on the use of central schemes for incompressible Navier-Stokes flows
}

\author{
R. Grauer ${ }^{1}$ and F. Spanier \\ Institute for Theoretical Physics I, Ruhr-Univesität Bochum, Germany
}

Key words: central-schemes, projection method, vorticity-stream function approach

Recently, central methods combined with ENO limiting [1], [2], [3] have become very popular for hyperbolic problems. The main advantage is the simplicity of this method since no Riemann problem has to be solved. The only information necessary to know from the system under consideration is an estimate of the spectral radius of the linearization of the flux, corresponding to the maximum wave speeds of the underlying system. Therefore, the method is attractive also for problems where the Riemann or approximate Riemann problem is too difficult to solve or to implement. This method has also been applied to the incompressible Navier-Stokes equation in two dimensions using the vorticity-stream function approach [4], [3]. This approach has been questioned by Nielsen and Naulin [5]. They compared the CWENO-scheme as introduced by Kurganov and Levy (section 5, example 5) [3] with a standard spectral scheme and a finite difference approach using the Arakawa [6] discretization. The comparison focused on the conservation of integral quantities as the total energy and the total enstrophy. Their conclusion was that the spectral and Arakawa scheme outperformed the CWENO scheme quite dramatically in respect of numerical dissipation. The result is that both the Arakawa and the spectral scheme converge to the true solution from above in respect to the global quantities (energy and enstrophy are too high if underresolved) whereas the CWENO scheme converges (more slowly) from below (energy and enstrophy are too low). Nielsen and Naulin [5] did not compare the amount of spurious oscillations where the CWENO proves to have much better properties.

Here, we demonstrate that simply switching from the stream-function approach to the integration of the primitive variables $\mathbf{u}$ with a projection method as discussed by Brown et al [9] reduces the numerical dissipation quite substan-

$\overline{1}$ The work was supported in part by the INTAS contract 00-292. 
tially so that the CWENO scheme approaches the properties of the spectral and the Arakawa scheme without producing oscillations near strong vortex sheets. For a second order scheme based on the primitive variables the absence of spurious oscillations has been demonstrated by Kupferman and Tadmor [7]. Concerning the amount of numerical operations for the two different formulations, it has to noted that for the vorticity/stream function formulation a Poisson equation equation for the streamfunction and the CWENO timestepping for the vorticity have to be solved, whereas for the formulation in the primitive variables a Poisson equation equation for the pressure and two CWENO steps for $u_{x}$ and $u_{y}$ have to be solved. It turns out the the time spent in the Poisson solver nearly equals the time spent in the CWENO step with the result that using the primitive variables is about 1.5 times slower than the vorticity/stream function formulation.

In the CWENO strategy there are two places where numerical dissipation is introduced: first by the ENO limiter which is the strongest source of dissipation and second by the averaging over the left and right approximations in the reconstruction step. The dissipation produced by this source can be minimized by sharpening the estimates of the maximum wave speeds. However, in the vorticity-stream function formulation vortex sheets have nearly a deltafunction like behavior in the vorticity whereas the primitive variables only develop steep gradients with almost bounded values of velocity. Therefore, it is more natural to work with the primitive variables then with vorticity. The primitive variables are integrated with the projection scheme similar to that of Kim and Moin [8] (see also Brown et al [9]) where the basic time step is listed for completeness:

$$
\begin{aligned}
& \frac{\mathbf{u}^{*}-\mathbf{u}^{n}}{\Delta t}+\left[((\mathbf{u} \cdot \nabla \mathbf{u})]^{n+1 / 2}=\nu \Delta \mathbf{u}^{n}\right. \\
& \Delta p=\frac{\nabla \cdot \mathbf{u}^{*}}{\Delta t} \\
& \mathbf{u}^{n+1}=\mathbf{u}^{*}-\Delta t \nabla p
\end{aligned}
$$

The integration in time is carried out with a standard low-memory third order Runge-Kutta step. For the range of high Reynolds numbers considered here we use an explicit treatment of the dissipation. The CWENO strategy is carried out for the velocity field $\mathbf{u}$ and the pressure $p$. Here, the treatment of the velocity is consistent as for the compressible Euler equations, whereas the velocity in the vorticity-stream function formulation is treated as if it where a passive quantity [3].

The difference of numerical dissipation for the CWENO treatment of the vorticity-stream function formulation and the integration of the primitive variables can clearly be seen in Figures 1 and 2 where we compare at a fixed time the evolution of two vortices as used by Nielsen and Naulin [5]. Figure 1 shows 
the result obtained by using CWENO with the vorticity stream function formulation whereas Figure 2 shows the same simulation using the primitive variables. It can clearly be seen that the maximum of vorticity is much better preserved and that the vortex spirals are much better resolved than in the vorticity stream function formulation. In addition, the spin up of the vortex arms differs in both calculations due to the different dissipation of the schemes. This has quite some impact on the global quantities as total energy and total enstrophy which are shown in Figure 3 and 4, respectively, for the two approaches. Simulation were carried out with two resolutions using $256^{2}$ and $512^{2}$ mesh points. As can be seen from the time evolution of the enstrophy, the vorticity-stream function simulation with $512^{2}$ mesh points reaches the quality of the integration of the primitive variables obtained with $256^{2}$ mesh points.

Another important advantage of the velocity-based scheme results from the easy combination with the penalty method [10], which will be discussed in a forthcoming paper.

We conclude that when applying the CWENO strategy to incompressible flows the formulation in primitive variables should be preferred.

\section{References}

[1] H. Nessyahu and E. Tadmor (1990), Non-oscillatory central differencing for hyperbolic conservation laws, J. Comput. Phys. 87408 (1990).

[2] A. Kurganov and E. Tadmor, (2000), New high-resolution central schemes for nonlinear conservation laws and convection-diffusion equations, J. Comp. Phys. 160214 (2000).

[3] A. Kurganov and D. Levy, A third-order semi-discrete central scheme for conservation laws and convection-diffusion equations, SIAM J. Sci. Computing 221461 (2000).

[4] D. Levy and E. Tadmor, Non-oscillatory central schemes for the incompressible 2-D Euler equations, Math. Res. Lett. 41 (1997).

[5] A. Nielsen and V. Naulin, Accuracy of spectral and finite difference schemes in 2D advection problems, to appear in SIAM Journal on Scientific Computing.

[6] A. Arakawa, Computational design for long-term numerical integration of the equations of fluid motion: Two-dimensional incompressible flow. part I, J. Comput. Phys., 1119 (1966).

[7] R. Kupferman and E. Tadmor, A fast high-resolution second-order central scheme for incompressible flows, Proc. Nat. Acad. Sciences 944848 (1997). 
[8] J. Kim and P. Moin, Application of a fractional-step method to incompressible Navier Stokes equations, J. Comput. Phys. 59, 308 (1985).

[9] D. L. Brown, R. Cortez and M. L. Minion, Accurate Projection Methods for the Incompressible Navier-Stokes Equation, J. Comput. Phys. 168464 (2001).

[10] E. A. Fadlun, R. Verzicco, P. Orlandi and J. Mohd-Yusof, Combined ImmersedBoundary Finite-Difference Methods for Three-Dimensional Complex Flow Simulation, J. Comput. Phys. 16135 (2000). 


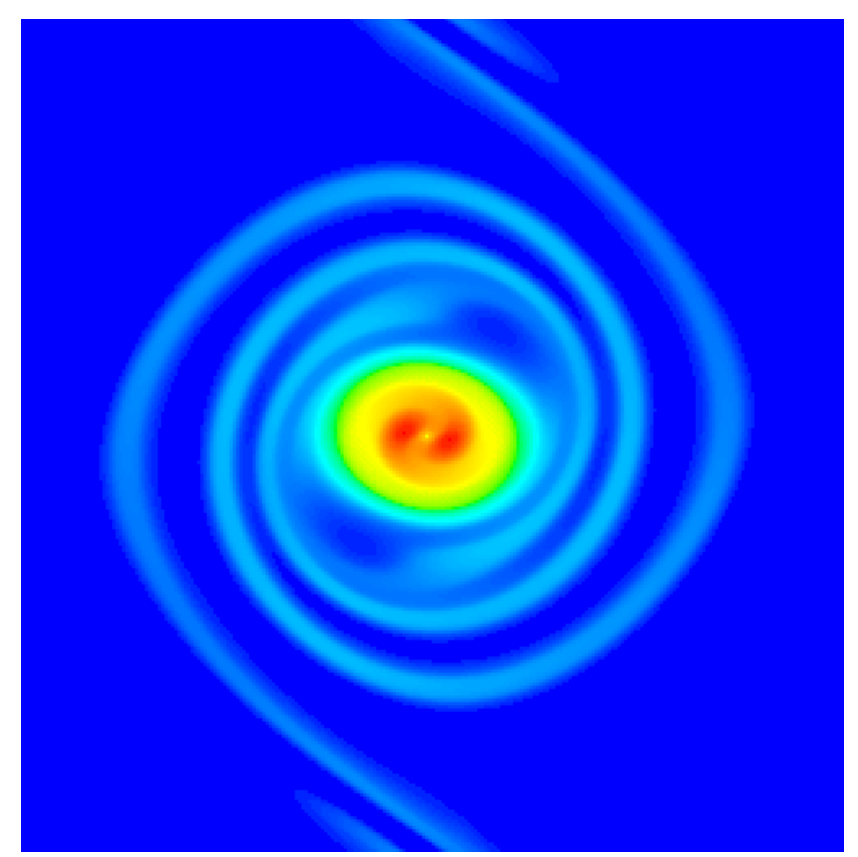

Fig. 1. Two Gauss-vortices with $R e=90000$ at time $t=10$, using vorticity-stream function (VS)-scheme

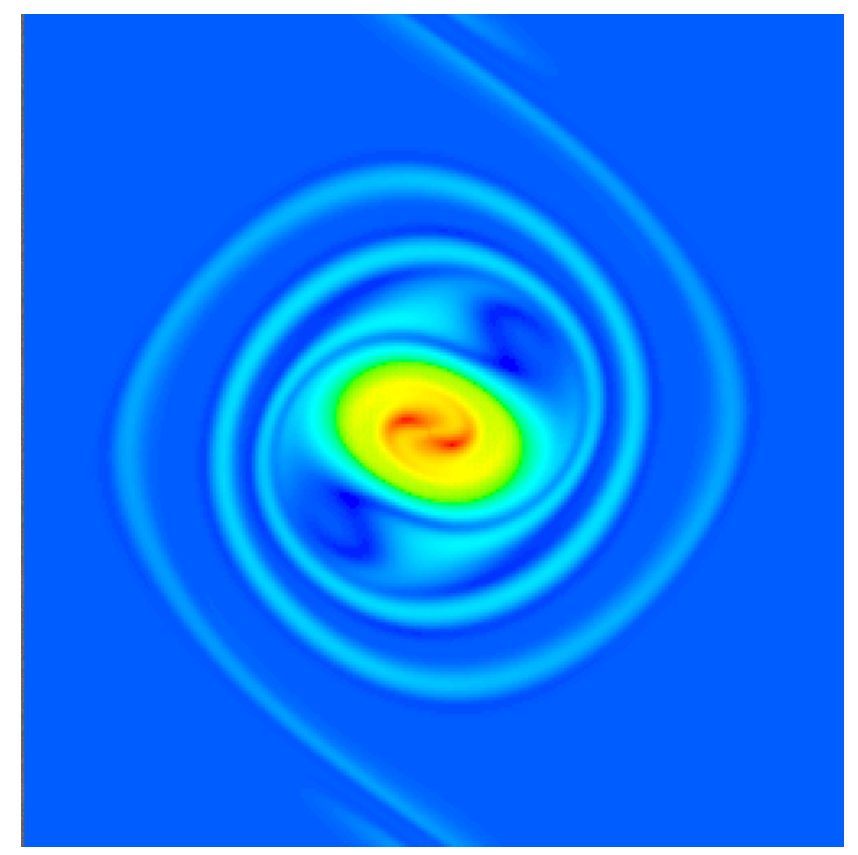

Fig. 2. Two Gauss-vortices with $R e=90000$ at time $t=10$, using PM II-scheme 


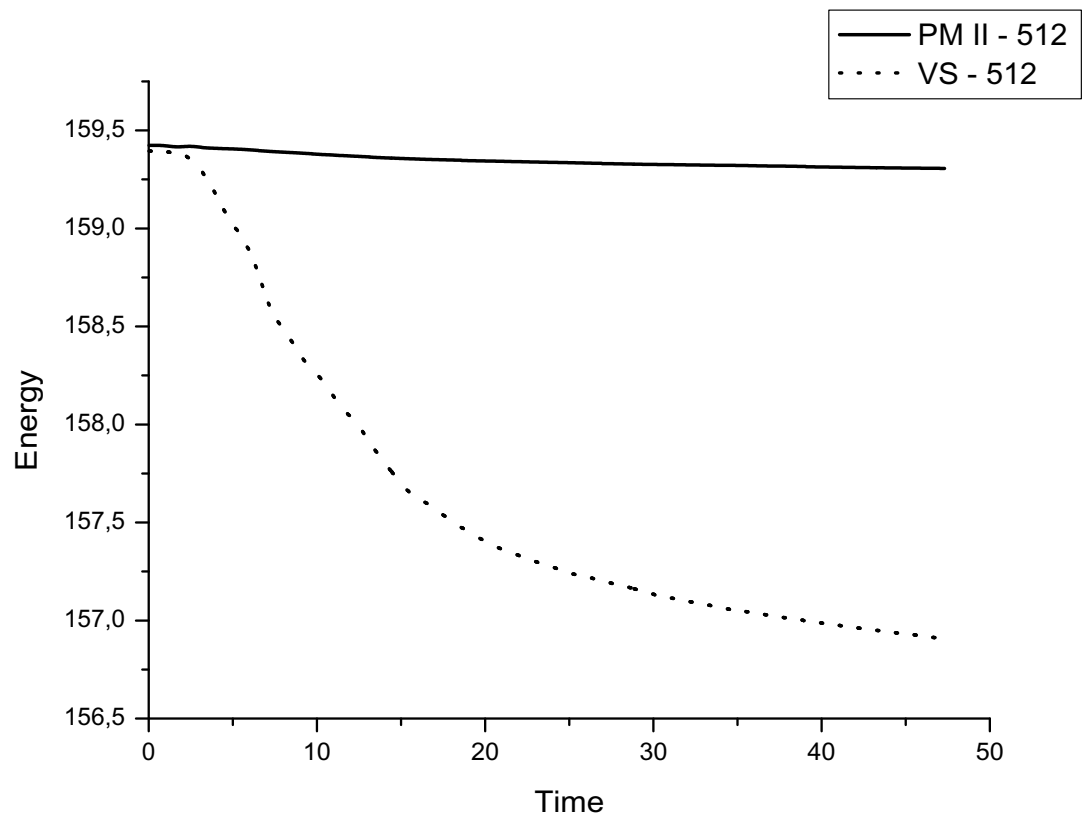

Fig. 3. Temporal evolution of enstrophy for both schemes, $R e=90000$

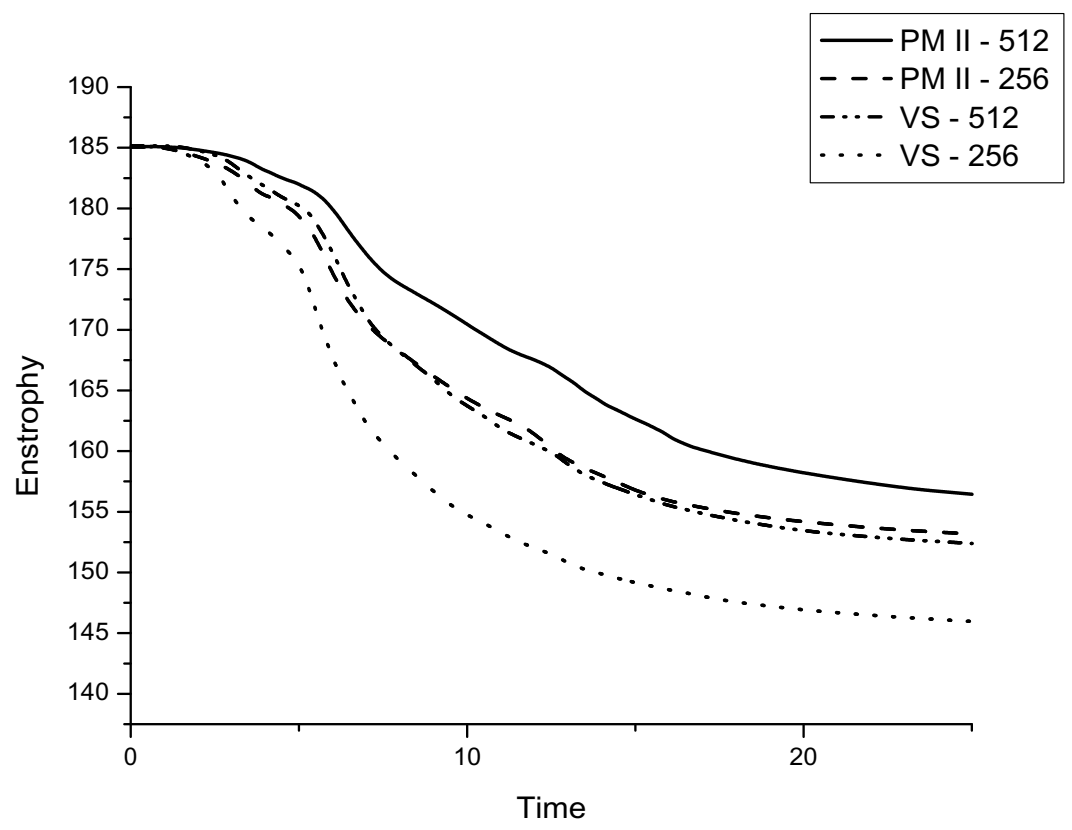

Fig. 4. Temporal evolution of enstrophy for both schemes, $R e=90000$ 